\title{
Pathogen burden, co-infection and major histocompatibility complex variability in the European badger (Meles meles)
}

\begin{abstract}
Pathogen-mediated selection is thought to maintain the extreme diversity in the major histocompatibility complex (MHC) genes, operating through the heterozygote advantage, rare-allele advantage and fluctuating selection mechanisms. Heterozygote advantage (i.e. recognizing and binding a wider range of antigens than homozygotes) is expected to be more detectable when multiple pathogens are considered simultaneously. Here, we test whether MHC diversity in a wild population of European badgers (Meles meles) is driven by pathogen-mediated selection. We examined individual prevalence (infected or not), infection intensity and co-infection of 13 pathogens from a range of taxa and examined their relationships with MHC class I and class II variability. This population has a variable, but relatively low, number of MHC alleles and is infected by a variety of naturally occurring pathogens, making it very suitable for the investigation of $\mathrm{MHC}$-pathogen relationships. We found associations between pathogen infections and specific MHC haplotypes and alleles. Co-infection status was not correlated with MHC heterozygosity, but there was evidence of heterozygote advantage against individual pathogen infections. This suggests that rare-allele advantages and/or fluctuating selection, and heterozygote advantage are probably the selective forces shaping MHC diversity in this species. We show stronger evidence for MHC associations with infection intensity than for prevalence and conclude that examining both pathogen prevalence and infection intensity is important. Moreover, examination of a large number and diversity of pathogens, and both MHC class I and II genes (which have different functions), provide an improved understanding of the mechanisms driving MHC diversity.
\end{abstract}

Keyword: Evolutionary arms race; Frequency-dependent selection; Heterozygote advantage hypothesis; Host-parasite co-evolution; Pathogen-mediated selection; Rare-allele advantage 